\title{
Disordered Nonlinear Metalens for Raman
}

\section{Spectral Nano-Imaging}

Sergey S. Kharintsev, ${ }^{1,2 *}$ Anton V. Kharitonov, ${ }^{1}$ Almaz R. Gazizov, ${ }^{1,2}$ and Sergei G. Kazarian ${ }^{3}$

${ }^{1}$ Department of Optics and Nanophotonics, Institute of Physics, Kazan Federal University, Kremlevskaya, 16, Kazan, 420008, Russia

${ }^{2}$ Institute of Applied Research, Tatarstan Academy of Sciences, Bauman str. 20, Kazan 420111, Russia.

${ }^{3}$ Department of Chemical Engineering, Imperial College London, SW7 2AZ, United Kingdom

*To whom correspondence should be addressed. E-mail: (S.S.K.) skharint@gmail.com

All optical experiments were performed on a commercial many-purpose analytical system NETRGA SPECTRA (NT-MDT). Fig. S1 shows a schematic of this setup combined with a metalens. A sample under study is directly positioned on the metalens surface. A $632 \mathrm{~nm}$ linearlypolarized (>100:1) laser light passes through a single-mode fiber is, then, filtered with a plasma line filter to suppress undesirable satellites. Tailoring to a lens aperture is performed with a beam expander that makes the laser beam of $4 \mathrm{~mm}$ in diameter. A polarization direction varies with a $\lambda / 2$ waveplate. After reflection from a notch filter, the collimated laser light is focused onto this sample with an 100x objective with a numerical aperture of 0.7 using the epi-configuration. A scattered light is collected with the same objective and moves towards the Cherny-Turner monochromator in which the light is decomposed into monochromatic components with a 600 
grooves/mm grating. Finally, the scattered light is detected with a water-cooled electron multiplying charge-coupled-device (EMCCD) (Newton, Andor) cooled to $-100^{\circ} \mathrm{C}$.

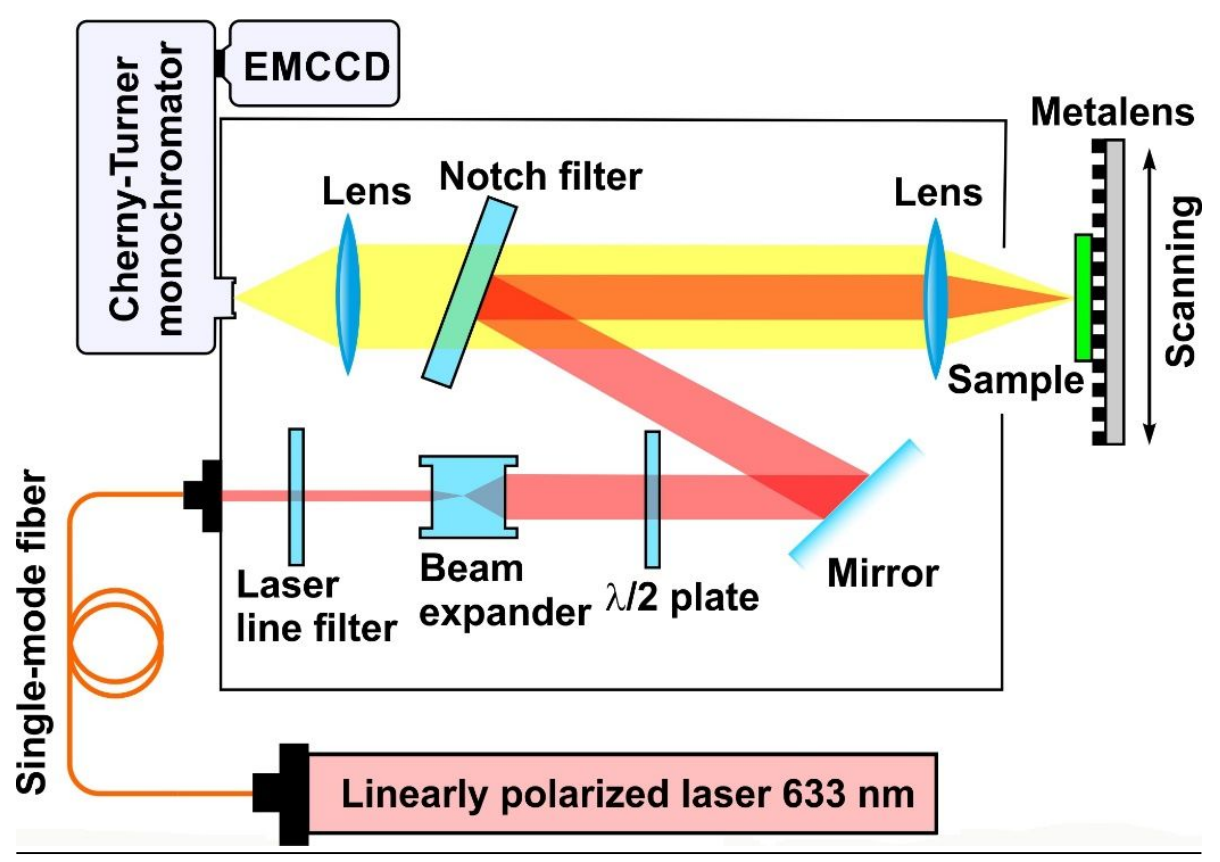

Figure S1. Schematic of an experimental setup.

Fig. S2 (a) shows Raman spectra of a $50 \mathrm{~nm}$ TiON thin film at different temperatures. The firstorder Raman peaks of $\mathrm{Si}$ at $520 \mathrm{~cm}^{-1}$ and $-520 \mathrm{~cm}^{-1}$ are utilized for plotting a temperature calibration Fig. S3 (b) using the following relationship:

$$
\frac{I_{a s}}{I_{s}}=\frac{\left(\omega_{0}+\Omega\right)^{4}}{\left(\omega_{0}-\Omega\right)^{4}} \exp \left[-\frac{h c \Omega}{k_{B} T}\right]
$$

where $\omega_{0}$ is the laser excitation frequency, $\Omega$ is a phonon frequency, $h$ is a Planck's constant, $c$ is the speed of light in vacuum, $k_{B}$ is a Boltzmann's constant and $T$ is the temperature at the sample. 



Figure S2. (a) Raman spectra of a $50 \mathrm{~nm}$ TiON thin film at different temperatures. (b) A plot of $\ln \left(I_{s} / I_{a s}\right)$ vs temperature.

Fig. S3 (a) shows an atomic force microscopy (AFM) image of a multi-walled carbon nanotube (MWCNT) placed on the TiN thin film. Raman spectra of the sample at $480 \mathrm{~cm}^{-1}$ and $1590 \mathrm{~cm}^{-1}$ are given in Fig. S3 (b) and (c), respectively. We see that the image of the MWCNT-loaded metalens is more blurred in contrast to the TiON metalens. Cross sections along a dashed straight line (1 and 2) proof the super-resolution of below than the diffraction limit (see Fig. S3). 

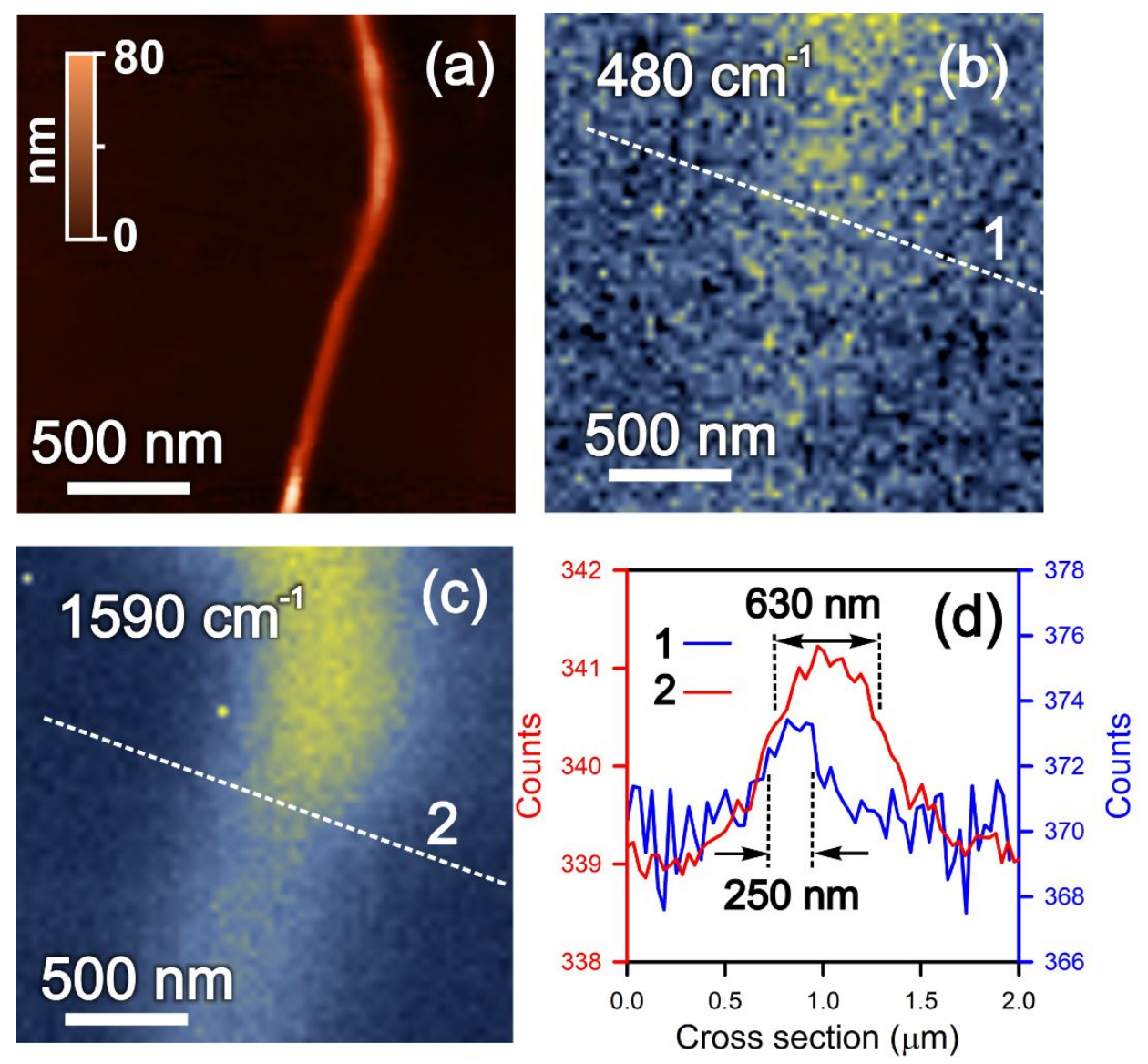

Figure S3. (a) Atomic force microscopy image of a multi-walled carbon nanotube positioned on a TiN thin film. (b) and (c) Raman maps of the MWCNT at $480 \mathrm{~cm}^{-1}$ (SRS) and $1590 \mathrm{~cm}^{-1}$ (graphite $G$-band), respectively, and (d) their cross sections along a dashed straight line.

Fig. S4 show far-field Raman images of the MWCNT on the disordered nonlinear TiON metalens at the different wavenumbers. It follows from this figure that the images at $1360 \mathrm{~cm}^{-1}$ and 1580 $\mathrm{cm}^{-1}$ are diffraction-limited $(550 \mathrm{~nm})$, whereas all other Raman images indicates a slightly better resolution (400 $\mathrm{nm})$, except the SRS overtones at 480, 720, 960 and $1200 \mathrm{~cm}^{-1}$ at which a superresolution in the far-field imaging is achieved. 

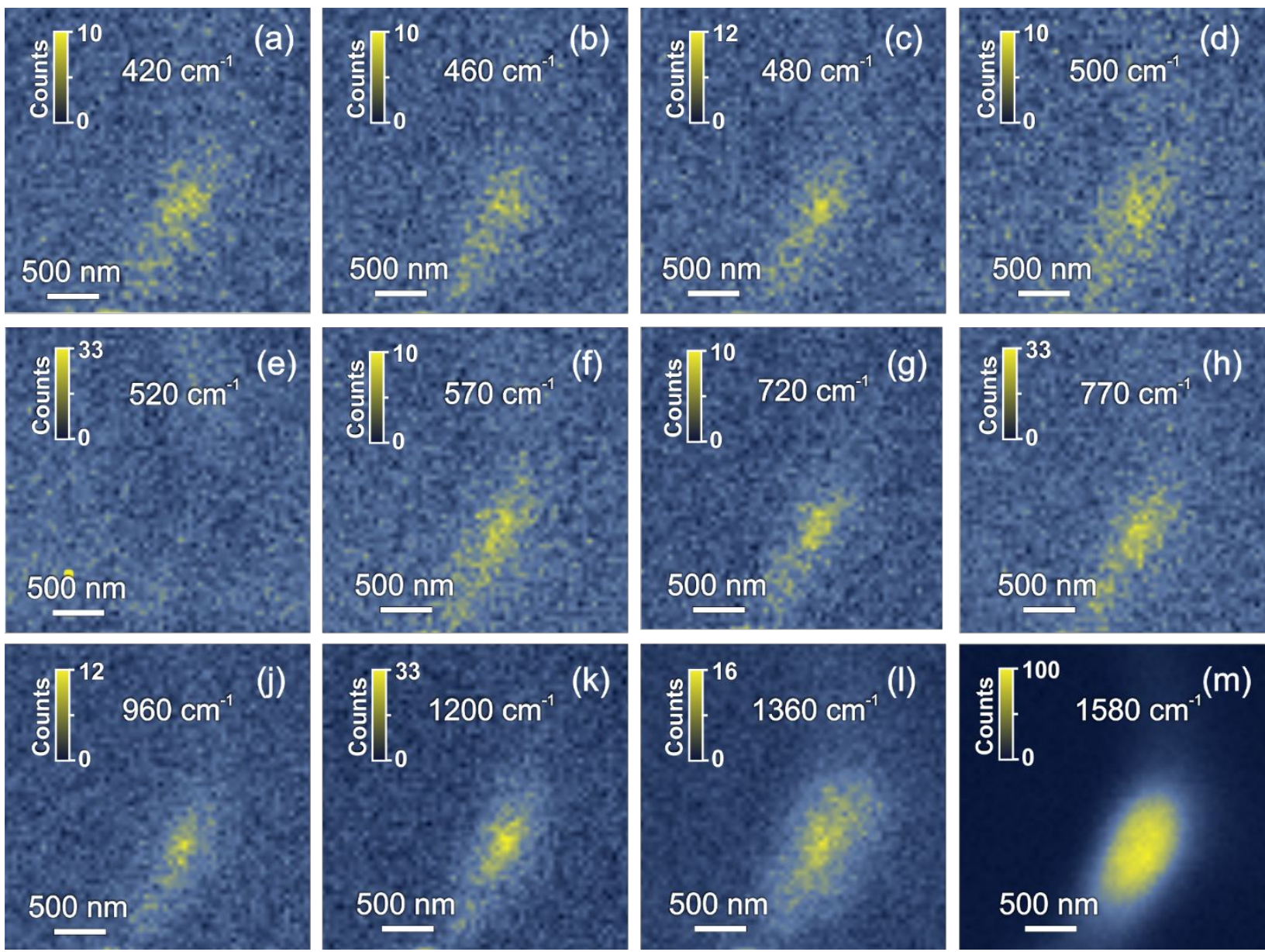

Figure S4. Far-field Raman imaging of the MWCNT on the TiON metalens at different wavenumbers (a)-(m). 